\title{
Regulatory Lymphocytes Are Key Factors in MHC-Independent Resistance to EAE
}

\author{
Nieves Marín,, ${ }^{1,2}$ Miriam Mecha, ${ }^{2,3}$ Carmen Espejo, ${ }^{2,4,5}$ Leyre Mestre, ${ }^{2,3}$ Herena Eixarch,,4,5 \\ Xavier Montalban, ${ }^{2,4,5}$ José C. Álvarez-Cermeño, ${ }^{1,5,6}$ Carmen Guaza, ${ }^{2,3}$ and Luisa M. Villar ${ }^{1,2}$ \\ ${ }^{1}$ Multiple Sclerosis Unit, Immunology and Neurology Departments, Hospital Universitario Ramón y Cajal, IRYCIS, \\ Carretera de Colmenar Km 9.100, 28034 Madrid, Spain \\ ${ }^{2}$ Red Española de Esclerosis Múltiple (REEM), RETICS, Fondo de Investigación Sanitaria, Instituto de Salud Carlos III, \\ Ministerio de Economía y Competitividad, Madrid, Spain \\ ${ }^{3}$ Neuroimmunology Group, Department of Functional and System Neurobiology, Instituto Cajal, \\ Consejo Superior de Investigaciones Científicas, Avenida Doctor Arce 37, 28002 Madrid, Spain \\ ${ }^{4}$ Servei de Neurologia-Neuroimmunologia, Centre d'Esclerosi Múltiple de Catalunya, Vall d'Hebron Institut de Recerca, \\ Hospital Universitari Vall d'Hebron, Passeig de la Vall d'Hebron 119-129, 08035 Barcelona, Spain \\ ${ }^{5}$ Universitat Autònoma de Barcelona, Bellaterra, 08193 Cerdanyola del Vallès, Spain \\ ${ }^{6}$ Department of Medicine, Universidad de Alcalá, Plaza de San Diego s/n, Alcalá de Henares, 28801 Madrid, Spain
}

Correspondence should be addressed to Luisa M. Villar; luisamaria.villar@salud.madrid.org

Received 1 December 2013; Revised 12 March 2014; Accepted 13 March 2014; Published 27 April 2014

Academic Editor: Silvia Sánchez-Ramón

Copyright (C) 2014 Nieves Marín et al. This is an open access article distributed under the Creative Commons Attribution License, which permits unrestricted use, distribution, and reproduction in any medium, provided the original work is properly cited.

\begin{abstract}
Background and Objectives. Resistant and susceptible mouse strains to experimental autoimmune encephalomyelitis (EAE), an inducible demyelinating experimental disease serving as animal model for multiple sclerosis, have been described. We aimed to explore MHC-independent mechanisms inducing resistance to EAE. Methods. For EAE induction, female C57BL/6 (susceptible strain) and CD1 (resistant outbred strain showing heterogeneous MHC antigens) mice were immunized with the 35-55 peptide of myelin oligodendrocyte glycoprotein $\left(\mathrm{MOG}_{35-55}\right)$. We studied $\mathrm{T}$ cell proliferation, regulatory and effector cell subpopulations, intracellular and serum cytokine patterns, and titers of anti-MOG serum antibodies. Results. Upon immunization with $\mathrm{MOG}_{35-55}$, T lymphocytes from susceptible mice but not that of resistant strain were capable of proliferating when stimulated with $\mathrm{MOG}_{35-55}$. Accordingly, resistant mice experienced a rise in regulatory B cells $(P=0.001)$ and, to a lower extent, in regulatory T cells $(P=0.02)$ compared with $\mathrm{C} 57 \mathrm{BL} / 6$ susceptible mice. As a consequence, $\mathrm{MOG}_{35-55}$-immunized $\mathrm{C} 57 \mathrm{BL} / 6$ mice showed higher percentages of CD4+ T cells producing both IFN-gamma $(P=0.02)$ and IL-17 $(P=0.009)$ and higher serum levels of IL-17 $(P=0.04)$ than resistant mice. Conclusions. Expansion of regulatory B and T cells contributes to the induction of resistance to EAE by an MHCindependent mechanism.
\end{abstract}

\section{Introduction}

Multiple sclerosis (MS) is a chronic inflammatory disease of the central nervous system (CNS). Numerous evidences strongly suggest that it is an autoimmune disease in which activated $\mathrm{T}$ cells enter the CNS and trigger an inflammatory cascade resulting in demyelination and axonal loss. Different genetic and environmental factors have shown to play a role in MS susceptibility, being class II alleles of the major histocompatibility complex (MHC), the more closely associated genes [1], and infectious agents such as EpsteinBarr virus, the environmental factors that have been more clearly associated with MS susceptibility [2]. Conversely, high serum levels of vitamin $\mathrm{D}$ have a protective role, delaying the appearance of a second demyelinating event after a clinically isolated syndrome [3]. It has been described that this action can be mediated by upregulation of regulatory $\mathrm{T}$ cells (Treg) [4] and B cells (Breg) [5]. It was also described that MS patients suffer from a peripheral $B$ cell tolerance defect that may be attributable to impaired Treg function [6] 
and that proliferation of Breg induced by intestinal helminth infections ameliorates MS course [7].

The role of regulatory cells in demyelinating diseases has been also explored in experimental autoimmune encephalomyelitis (EAE), a widely used animal model of MS in which T cell mediates CNS demyelination [8]. EAE can be induced in several species by immunization with myelin antigens or via adoptive transfer of myelin-reactive T cells $[9,10]$. Adoptive transfer of Breg reduced significantly the severity of EAE by inhibiting Thl and Thl7 inflammatory responses [11]. Likewise, expansion of Treg diminished the infiltration of inflammatory cells into the CNS and improved clinical signs of EAE [12]. These data strongly suggest that deficits in regulatory pathways may contribute to the induction of autoimmune reactivity in EAE. These experimental models may be useful tools to identify immunological mechanisms mediating a protective role in demyelinating diseases.

Several strains of mice are resistant to develop EAE as mice are asymptomatic following immunization with myelin antigens [13]. It was argued that a poor recognition of encephalitogenic peptides by MHC molecules might contribute to resistance to EAE. However, other immune mechanisms may also contribute to inducing resistance to the disease. We explored them in two mouse strains, CD1 and C57BL/6, that are, respectively, resistant and susceptible to EAE induced by the myelin oligodendrocyte glycoprotein 3555 peptide $\left(\mathrm{MOG}_{35-55}\right)$. Since $\mathrm{CD} 1$ is an outbred strain, these mice do not share a unique MHC haplotype, which makes this model useful to study MHC-independent mechanisms that mediate resistance to this experimental model of MS. We explored Breg and Treg in both strains and studied if variations in regulatory cells associated with downregulation of effector immune mechanisms.

\section{Materials and Methods}

2.1. Mice. Eight-to-ten-week-old female C57BL/6J mice, which are susceptible to EAE induction by $\mathrm{MOG}_{35-55} \mathrm{immu-}$ nization, were purchased from Harlan (Barcelona, Spain). Age- and sex-matched CD1 mice that are resistant to EAE induction were obtained from our own conventional barrier protection breeding colony (Cajal Institute, CSIC, Madrid, Spain). Mice were housed under standardized light- and climate-controlled conditions and were fed with standard chow and water ad libitum. Experiments were done in compliance with the guidelines of animal care set by the European Union (86/609/EEC), and all animal protocols were approved by the Cajal Institute animal welfare committee (Protocol N IORG0006540).

2.2. EAE Induction. For immunization C57BL/6 and CD1 mice were injected subcutaneously on day 0 with $200 \mu \mathrm{L}$ of an emulsion containing $300 \mu \mathrm{g}$ of $\mathrm{MOG}_{35-55}$ (CNB, CSIC, Madrid, Spain) and $800 \mu \mathrm{g}$ of heat killed Mycobacterium tuberculosis H37RA (Difco, BD Diagnostics, MD) emulsified in incomplete Freund adjuvant oil (Sigma-Aldrich, St. Louis, MO). In addition, the mice received $100 \mathrm{ng}$ of Bordetella pertussis toxin (Sigma-Aldrich) intraperitoneally on days 0 and 2 after immunization (p.i.). Mice from both strains C57BL/6 or CD1 were immunized in the same way using the 139-151 peptide of proteolipid protein $\left(\mathrm{PLP}_{139-151}\right)$ (Proteomics Section, Universitat Pompeu Fabra, Barcelona, Spain) for comparison with $\mathrm{MOG}_{35-55}$ as C57BL/6 mice do not develop EAE following immunization with PLP peptide. Age-matched naïve C57BL/6 or CD1 mice were considered as control groups.

Mice were monitored daily, and clinical signs of EAE were graded as follows: Grade 0, normal; Grade 1, flaccid tail; Grade 2, mild hind-limb weakness; Grade 3, severe hind-limb weakness; Grade 4, hind-limb paralysis; Grade 5, hind-limb paralysis and partial fore-limb weakness.

2.3. Experimental Procedure. On the basis of previous reports [14] we selected day 21 p.i. as the time point in which experimental procedures were performed. Mice were anaesthetized by intraperitoneal administration of EuTalender (Normonlab, Madrid, Spain). Blood samples were collected by intracardiac puncture. Serum was obtained and frozen at $-80^{\circ} \mathrm{C}$ until soluble cytokines and antibodies were assayed. Spleens were removed and splenocyte suspensions were generated by grinding spleens through a wire mesh. Lymphocyte subsets and intracellular cytokine production were tested in freshly isolated splenocytes.

2.4. Splenocyte Proliferation Assay. The splenocytes were seeded in 96-well plates at a cell density of $2 \times 10^{5}$ cells/well in Iscove's modified Dulbecco's medium (IMDM; PAA Laboratories $\mathrm{GmbH}$, Pasching, Austria) supplemented with 10\% HyClone FetalClone I (Thermo Fisher Scientific, Waltham, MA, USA), $50 \mu \mathrm{mol} / \mathrm{L}$ of 2-mercaptoethanol (Sigma Chemical), $2 \mathrm{mmol} / \mathrm{L}$ of glutamine, $50 \mathrm{U} / \mathrm{mL}$ of penicillin, and $50 \mathrm{mg} / \mathrm{mL}$ of streptomycin; the last three chemicals were obtained from Gibco BRL (Paisley, UK). For splenocyte activation, we used $5 \mu \mathrm{g} / \mathrm{mL}$ of $\mathrm{MOG}_{35-55}, \mathrm{PLP}_{139-151}$, or phytohaemagglutinin (PHA; Sigma Chemical). Cells were incubated in a humidified atmosphere at $5 \% \mathrm{CO}_{2}$ and $37^{\circ} \mathrm{C}$ for 3 days, the last $18-20 \mathrm{~h}$ in the presence of $1 \mu \mathrm{Ci} /$ well of $\left[{ }^{3} \mathrm{H}\right]$ thymidine (PerkinElmer Inc., USA). The levels of incorporated radioactivity were determined using a beta-scintillation counter (Wallac, Turku, Finland). The stimulation index (SI) was expressed as the mean of the counts per minute (cpm) of five replicates from each mouse and culture condition divided by the mean cpm of the baseline control replicates. The results are expressed as the mean value \pm standard error of the mean (SEM) of the SI per group of mice.

2.5. Study of B and T Cell Subsets. We performed this study in series of seven mice per condition. To study lymphocyte subsets, cells were resuspended at $10^{6} / \mathrm{mL}$ and incubated with rat anti-mouse $\mathrm{CD} 16 / 32 \mathrm{Fc}$ receptor $(\mathrm{FcR})(\mathrm{CD} 16 / \mathrm{CD} 32)$ during 10 minutes to avoid unspecific staining. Then, they were incubated with the appropriate combinations of monoclonal antibodies (BD Pharmingen, San Diego, CA) for 20 minutes at $4^{\circ} \mathrm{C}$. Cells were washed with PBS and flow-cytometry analysis was performed on FACScanto II (Becton Dickinson) and analyzed using the DIVA software (Becton Dickinson). As strategy for flow-cytometry analysis, an initial region 
was set around cells expressing intermediate to high CD45 with low-to-intermediate side scatter (P1) and then a second region was set on the forward/side scatter dot plot to exclude debris or apoptotic cells (P2). Only cells included in P1 and $\mathrm{P} 2$ regions were accepted for analysis. We used the following monoclonal antibodies: anti-CD4-FITC, anti-CD8a-PE, antiCD45-Cy5.5PerCP, anti-CD25-APC, anti-B220-FITC, antiCD1d-PE, and anti-CD5-APC, and isotype controls conjugated with FITC, PE, Cy5.5PerCP, and APC.

2.6. Analysis of Intracellular Cytokine Production. Splenocytes were resuspended $\left(10^{6}\right.$ cells $\left./ \mathrm{mL}\right)$ in complete medium [RPMI supplemented with $2 \mathrm{mM}$ glutamine, $2 \mathrm{mg} / \mathrm{mL}$ gentamycin (all from Gibco BRL (Paisley, UK)), and 10\% fetal calf serum (Biowhittaker technologies, MD, USA)] and the cells were cultured for four hours with PMA $(50 \mathrm{ng} / \mathrm{mL}$; SigmaAldrich, St. Louis, MO), ionomycin $(500 \mathrm{ng} / \mathrm{mL}$; SigmaAldrich), and monensin ( $2 \mu \mathrm{M}$; eBioscience, San Diego, CA $)$.

For IL-17A and IFN-gamma detection, FcR were blocked with anti-CD16/CD32 antibody (BD Pharmingen) for 10 minutes at room temperature before cell surface staining. Cells were stained with antibodies against surface antigens for $30 \mathrm{~min}$ at $4^{\circ} \mathrm{C}$ using predetermined optimal concentrations of each antibody. Stained cells were fixed and permeabilized using a Cytofix/Cytoperm kit (BD Pharmingen), according to the manufacturer's instructions, and stained with anti-IL17A-PE and anti-IFN-gamma-APC for $30 \mathrm{~min}$ at $4^{\circ} \mathrm{C}$. After washing cells, flow-cytometry analysis was performed on FACScanto II (Becton Dickinson) and analyzed using the DIVA software (Becton Dickinson).

2.7. Quantification of Serum Cytokines. Serum cytokines were determined by a Milliplex map mouse cytokine panel (Millipore, Billerica, MA) following manufacturer instructions. Data were analyzed in a TM100 cytometer (Labscan, Gliwice, Poland).

2.8. Detection of Anti-MOG ${ }_{35-55}$ Antibodies. Maxisorp microtiter plates (Nunc, Roskilde, Denmark) were coated overnight at $4^{\circ} \mathrm{C}$ with $100 \mu \mathrm{L} /$ well of mouse $\mathrm{MOG}_{35-55}$ peptides (CNB, CSIC) at a concentration of $10 \mu \mathrm{g} / \mathrm{mL}$ in $0.1 \mathrm{M}$ carbonate buffer, $\mathrm{pH}$ 9.7. Plates were washed with PBS containing $0.02 \%$ Tween 20 and blocked with $0.1 \mathrm{M}$ carbonate buffer, $\mathrm{pH} 9.7$, containing 5\% BSA for $1 \mathrm{~h}$ at $37^{\circ} \mathrm{C}$. Then, plates were incubated with triplicate serum samples diluted at 1/200 for $1 \mathrm{~h}$ at $37^{\circ} \mathrm{C}$ and washed and incubated with biotinylated anti-mouse IgM (Sigma-Aldrich) or anti-mouse IgG (Jackson Immunoresearch, Suffolk, UK) for $1 \mathrm{~h}$ at $37^{\circ} \mathrm{C}$. After this, they were washed and incubated with streptavidin-horseradish peroxidase (Roche, Basel, Switzerland) for $30 \mathrm{~min}$ at $37^{\circ} \mathrm{C}$. After a final wash, the reaction products were visualized using OPD (Sigma-Aldrich) as a substrate and read at $492 \mathrm{~nm}$ with a microplate reader (Thermo Fisher, Cambridge, UK).

2.9. Statistical Analysis. Results were analyzed with Prism 5.0 statistical package. We used Mann-Whitney $U$ test for comparisons between groups. $P$ values lower than 0.05 were considered significant.
Experiments were always performed in two independent series of mice using four animals per condition. We combined them for the final statistical analysis. Results are always expressed as mean \pm SEM.

\section{Results}

We explored regulatory and effector immune mechanisms in $\mathrm{CD} 1$ and $\mathrm{C} 57 \mathrm{BL} / 6$ mice immunized with $\mathrm{MOG}_{35-55}$, using as controls nonimmunized mice and mice immunized with PLP $_{139-151}$, a myelin peptide that is not capable of inducing EAE in any of those mouse strains.

3.1. Splenocyte Proliferative Response. We tested the polyclonal and antigen-specific proliferative capacity of splenocytes 21 days after the immunization. A diminished proliferation showing a clear statistical trend $(P=0.056)$ was detected in the resistant strain when a polyclonal stimulus such as PHA was used (Figure 1). Moreover, upon antigenspecific stimulation, the splenocytes from CD1 resistant mice did not proliferate, while those of C57BL/6 susceptible mice did $(P=0.016$, Figure 1). Control study using irrelevant $\mathrm{PLP}_{139-151}$ peptide as stimulus did not render proliferation in any of the strains. All these data indicate that despite their heterogeneous MHC antigens, all CD1 resistant mice are unable to develop a $\mathrm{T}$ cell response against the $\mathrm{MOG}_{35-55}$ encephalitogenic peptide. We next aimed to explore immunological mechanisms that contribute to this phenomenon.

3.2. B Cell Subsets. We first examined B1 and B2 subsets (Figures 2(a) and 2(b)). We did not found differences in the percentages of $\mathrm{T}$ cell dependant B2 cells (B220+CD5-) between resistant and susceptible mice (nonimmunized or immunized with MOG or PLP peptides). Conversely, the percentage of innate $\mathrm{B} 220+\mathrm{CD} 5+\mathrm{B}$ cells was downmodulated in C57BL/6 mice after EAE induction by $\mathrm{MOG}_{35-55}(P=0.005)$ when compared with $\mathrm{CD1}$ resistant mice immunized with the same peptide. These differences were due to the regulatory B220+CD5+CD1d ${ }^{\text {hi }}$ B cell subset (Breg). When exploring these $\mathrm{B}$ cells, we detected a decrease of Breg in C57BL/6 mice suffering $\operatorname{EAE}(P=0.02$, Figures $2(\mathrm{c})$ and $2(\mathrm{~d}))$. Conversely, resistant $\mathrm{CD} 1$ strain upregulated this subpopulation after immunization with $\mathrm{MOG}_{35-55}(P=0.03)$. These differences between the two strains $(P=0.001)$ were only observed upon $\mathrm{MOG}_{35-55}$ immunization. By contrast, Breg remained unchanged in either resistant or susceptible mice immunized with the PLP ${ }_{139-151}$ irrelevant peptide. This strongly suggests that Breg are specifically triggered to prevent the autoimmune process and have an important role in inducing resistance to EAE.

3.3. Regulatory $T$ Cells. Breg may contribute to the differentiation of regulatory $\mathrm{T}$ cells (Treg) [15]. We also explored this $\mathrm{T}$ cell subset $\left(\mathrm{CD} 4+, \mathrm{CD} 25^{\mathrm{hi}}\right)$ in our series of mice (Figure 3). Upon immunization with $\mathrm{MOG}_{35-55}$ resistant but not susceptible mice upregulated Treg $(P=0.028)$. No differences were found in this $\mathrm{T}$ cell subset between 
TABLE 1: Titers of anti-MOG serum antibodies and T cell and B cell percentages in spleen of mice resistant (CD1) and susceptible (C57BL/6) to $\mathrm{MOG}_{35-55}$ induced EAE.

\begin{tabular}{|c|c|c|c|c|c|c|c|c|c|}
\hline & \multicolumn{3}{|c|}{ Nonimmunized } & \multicolumn{3}{|c|}{$\mathrm{MOG}_{35-55}$-immunized } & \multicolumn{3}{|c|}{ PLP $_{139-151}$-immunized } \\
\hline & $\mathrm{C} 57 \mathrm{BL} / 6$ & CD1 & $P$ & C57BL/6 & CD1 & $P$ & $\mathrm{C} 57 \mathrm{BL} / 6$ & CD1 & $P$ \\
\hline MOG-IgG $\left(\mathrm{OD}_{492 \mathrm{~nm}}\right)$ & $0.24 \pm 0.02$ & $0.17 \pm 0.07$ & 0.59 & $0.12 \pm 0.03$ & $0.16 \pm 0.03$ & 0.34 & $0.27 \pm 0.07$ & $0.07 \pm 0.03$ & 0.11 \\
\hline MOG-IgM $\left(\mathrm{OD}_{492 \mathrm{~nm}}\right)$ & $0.15 \pm 0.03$ & $0.06 \pm 0.04$ & 0.10 & $0.12 \pm 0.07$ & $0.05 \pm 0.02$ & 0.62 & $0.04 \pm 0.01$ & $0.06 \pm 0.03$ & 0.47 \\
\hline$\%$ CD $4+\mathrm{T}$ cells & $17.8 \pm 0.96$ & $13.5 \pm 1.63$ & 0.11 & $17.04 \pm 3.22$ & $12.8 \pm 3.12$ & 0.48 & $19.8 \pm 3.33$ & $11.5 \pm 1.79$ & 0.11 \\
\hline \% CD8+ T cells & $27.4 \pm 1.53$ & $20.3 \pm 2.78$ & 0.11 & $28.0 \pm 3.30$ & $24.3 \pm 1.69$ & 0.34 & $21.5 \pm 1.02$ & $18.0 \pm 3.34$ & 0.34 \\
\hline
\end{tabular}

We studied nonimmunized mice and mice immunized with $\mathrm{MOG}_{35-55}$ and $\mathrm{PLP}_{139-151}$. We used eight animals per condition. Values are expressed as mean \pm standard error. No differences were found in the levels of IgM or IgG anti-MOG $\mathrm{MO}_{35-55}$ antibodies expressed as optical density at $492 \mathrm{~nm}$, in the percentages of $\mathrm{CD} 4+$ and CD8+ T lymphocytes, and total B cells (B220+) between the different experimental groups.

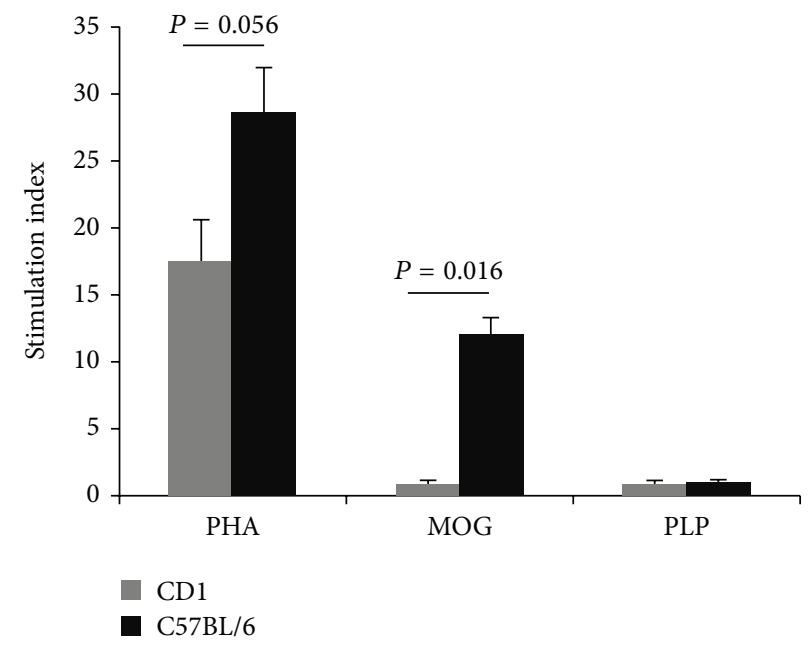

FIGURE 1: Proliferative capacity of splenocytes from C57BL/6 (susceptible strain) and CD1 (resistant strain) mice. We analyzed proliferative capacity in series of seven mice per condition. Splenocytes from resistant and susceptible mice were stimulated with a polyclonal stimulus (PHA), $\mathrm{MOG}_{35-55}$, or PLP $\mathrm{PL}_{139-151}$. Susceptible mice showed a higher proliferative capacity upon PHA stimulation and a statistically significant augmented proliferation upon MOG stimulation. Conversely, splenocytes from both strains did not proliferate when the stimulus used was 139-151 PLP peptide.

resistant and susceptible strains when nonimmunized mice or mice immunized with the $\mathrm{PLP}_{139-151}$ peptide were studied. These observations indicate that Treg may also play a role in resistance to EAE shown by CD1 mice immunized with $\mathrm{MOG}_{35-55}$.

3.4. Effector Responses. We did not find differences in the levels of IgM or IgG anti-MOG $\mathrm{MO}_{35-55}$ antibodies or in total $\mathrm{CD} 4+$ and $\mathrm{CD} 8+\mathrm{T}$ cell percentages between the resistant and the susceptible mice (Table 1). To further discriminate different $\mathrm{T}$ cell responses, we analyzed Th1 and Th17 subsets. We explored the percentage of CD4+ T cells showing intracellular production of IFN-gamma (Figure 4) and IL17 (Figure 5) upon stimulation with phorbol-12-myristate-13acetate (PMA) and ionomycin. Only susceptible mice that developed EAE in response to $\mathrm{MOG}_{35-55}$ showed significant increases in the secretion of IFN-gamma $(P=0.02)$ and IL-17 $(P=0.009)$ compared to resistant mice. By contrast, resistant mice were unable to mount any of these responses after the same immunization protocol. In addition, when studying serum inflammatory cytokines, we detected a significant increase of IL-17 in susceptible mice immunized with
$\mathrm{MOG}_{35-55}$ (Figure 4(c)). This shows that downmodulation of regulatory $\mathrm{B}$ and T cells is related to the induction of Th1/Th17 responses in MOG-induced EAE.

\section{Discussion}

The understanding of the immune mechanisms involved in resistance/susceptibility to EAE is important, as it may contribute to identification of new therapeutic targets in MS. The role of MHC by inducing defective or appropriate presentation of the encephalitogenic peptide has been previously demonstrated [16]. However, different evidences suggest that induction of Breg may also contribute to the resistance to EAE. They are necessary to induce Treg mediated recovery from EAE [15]. Moreover, depletion of Breg exacerbates EAE symptoms and increases encephalitogenic $\mathrm{T}$ cell influx into the CNS [11]. In addition, induction of Breg in MOG-immunized-C57BL/6 mice treated with antibiotics ameliorates EAE course, and this effect can be transferred to other $\mathrm{C} 57 \mathrm{BL} / 6$ mice by passive transfer of these $\mathrm{B}$ cells. Here, we aimed to explore if Breg may be a $\mathrm{MHC}$-independent mechanism for inducting resistance to 


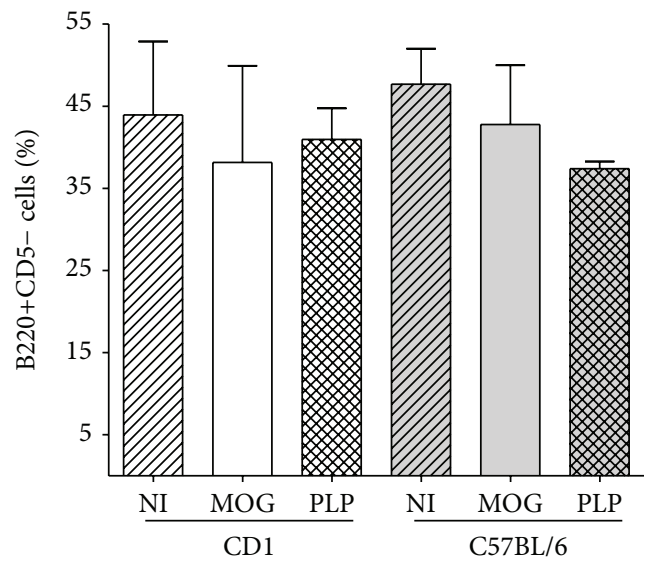

(a)

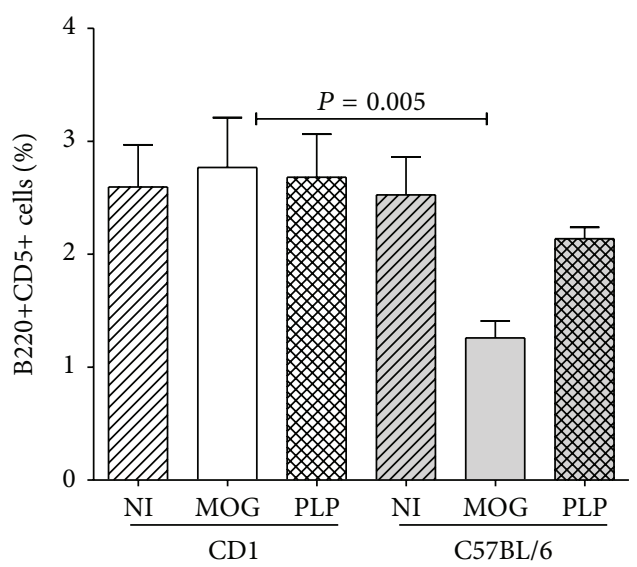

(b)

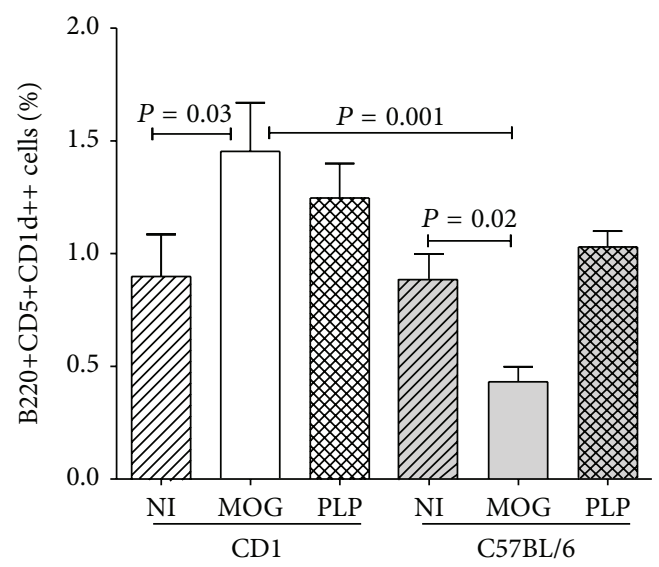

(c)
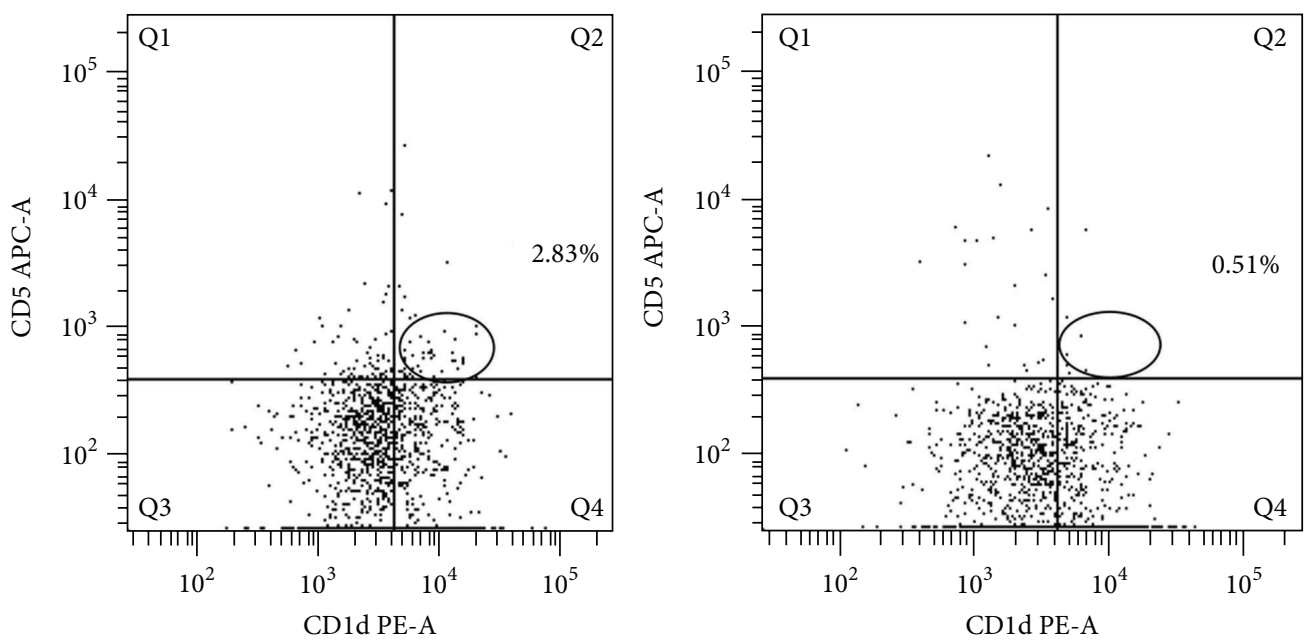

(d)

Figure 2: Effector and regulatory B cells in spleens of C57BL/6 and CD1 mice. We analyzed these cells in series of seven mice per condition. (a) B2 cells (B220+CD5-) did not change significantly in mice immunized with $\mathrm{MOG}_{35-55}$ (MOG) or PLP ${ }_{139-151}$ (PLP) when compared with nonimmunized (NI) ones. (b) B1 cell (B220+CD5+) percentage diminished in C57BL/6 mice that developed EAE upon immunization with $\mathrm{MOG}_{35-55}$. (c) The B220+CD5+CD1d ${ }^{\text {hi }}$ regulatory subset (Breg) increased in resistant CD1 mice immunized with MOG. Conversely, it decreased in susceptible C57BL/6 mice developing EAE after immunization with the same peptide. (d) Representative dot plots showing Breg induced in CD1 (left side) and C57BL/6 (right side) mice upon immunization with $\mathrm{MOG}_{35-55}$ peptide. Total B cells were gated by selecting B-220 positive cells and then Breg were identified by CD5 and CD1d ${ }^{\text {hi }}$ staining. 


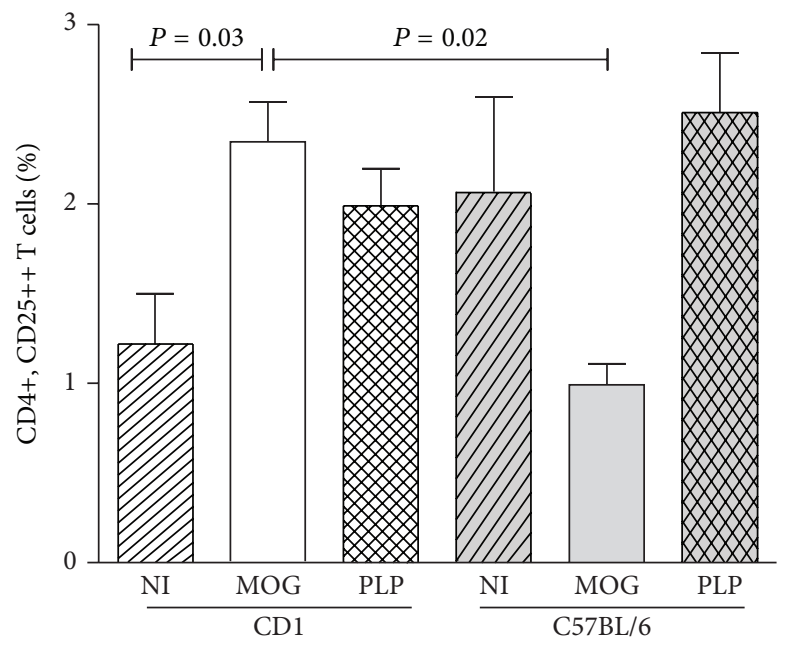

(a)
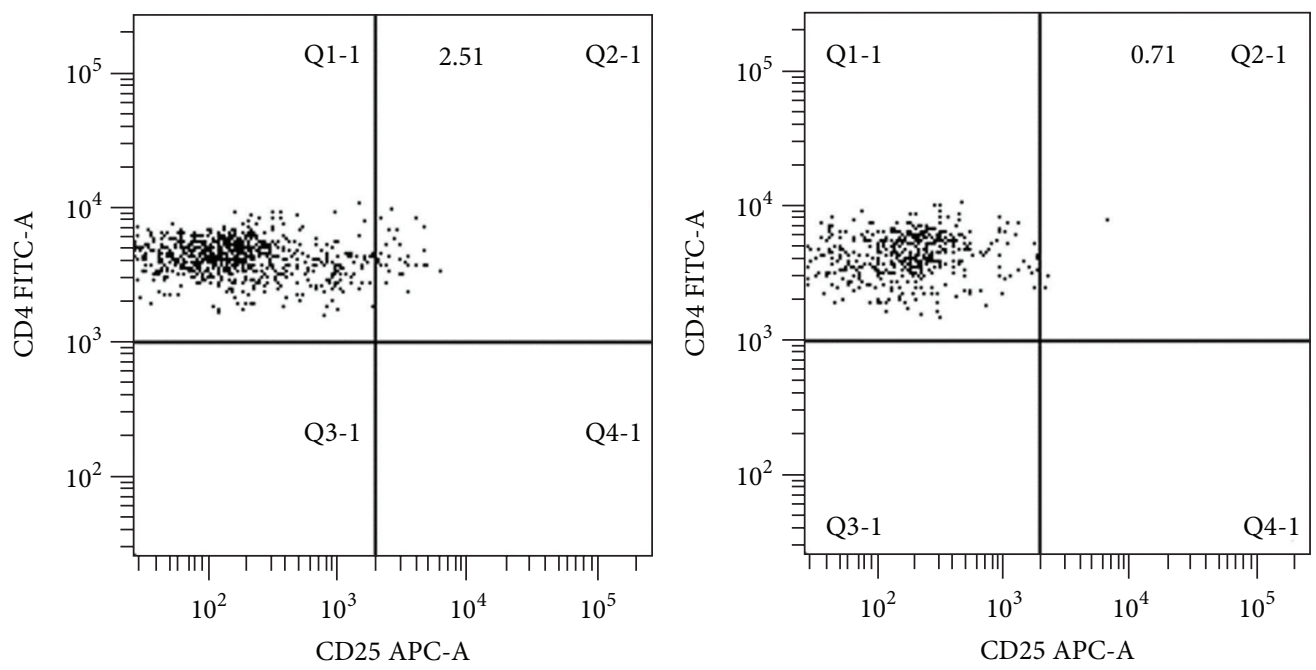

(b)

FIgURE 3: Regulatory T cells in spleens of resistant and susceptible mice. We analyzed these cells in series of seven mice per condition.

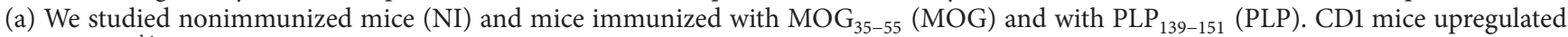
$\mathrm{CD} 4+\mathrm{CD} 25^{\text {hi }} \mathrm{T}$ cells when immunized with MOG or PLP autopeptides. Conversely, Treg percentages did not increase in C57BL/6 mice immunized with MOG that developed EAE. (b) Representative dot plots showing regulatory T cells induced in CD1 (left side) and C57BL/6 (right side) mice upon immunization with $\mathrm{MOG}_{35-55}$ peptide.

MOG-induced EAE. We explored this in C57BL/6, a congenic mouse strain susceptible to $\mathrm{MOG}_{35-55}$-induced EAE, and in $\mathrm{CD} 1$ an outbred strain resistant to $\mathrm{MOG}_{35-55}$-induced EAE in an MHC-independent manner. We immunized both strains of mice with the encephalitogenic peptide and observed that $\mathrm{CD} 1$ splenocytes were incapable of proliferating when stimulated with $\mathrm{MOG}_{35-55}$. We next explored immunological mechanism associated with this phenomenon and observed that susceptible C57BL/6 mice developing the disease after $\mathrm{MOG}_{35-55}$ immunization downregulate Breg, while CD1 resistant mice show a significant increase of these cells upon immunization with the same peptide. This strongly suggests that Breg play a role in the induction of resistance to EAE in CD1 mice.

Breg may regulate inflammatory responses by themselves and by inducing Treg [15]. It was recently shown that Treg are less suppressive in patients with MS [17]. In addition, an increase of Treg may prevent the onset of EAE [18]. In the present study we found that, in parallel with Breg findings, resistant mice upregulated Treg. This seems to indicate that Treg also have a role in resistance to MOG-induced EAE.

However, the immunization of C57BL/6 mice with the $\mathrm{PLP}_{139-151}$ peptide, which is incapable of inducing EAE in this strain in an MHC-dependent manner [19], did not induce significant changes in Breg or Treg subsets. We found no significant changes in both Breg and Treg. This clearly shows that when MHC molecules impede the correct peptide presentation, regulatory responses are not triggered as it happens in the outbred mice.

To further study differences between resistant and susceptible mice, we explored effector responses in the same series of mice. It has been reported that antibodies may have 


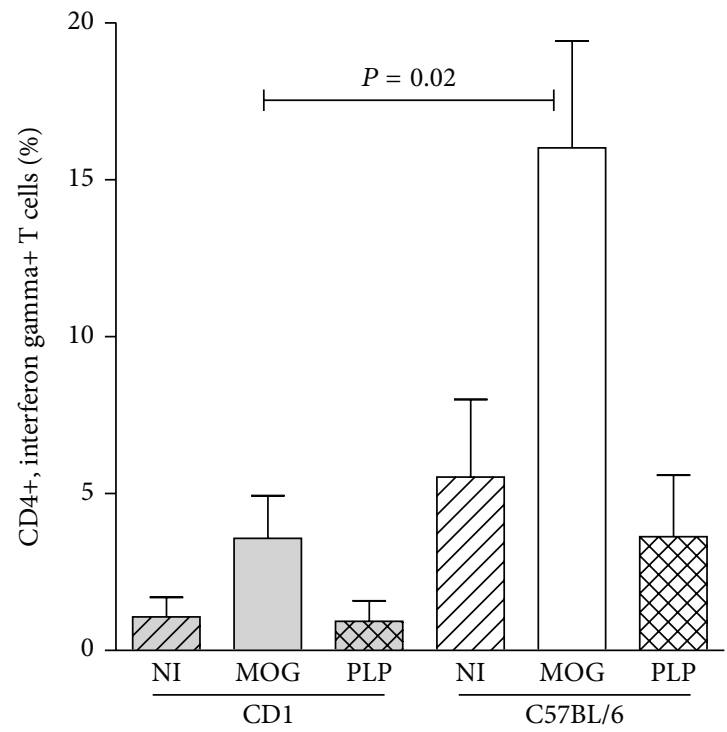

(a)
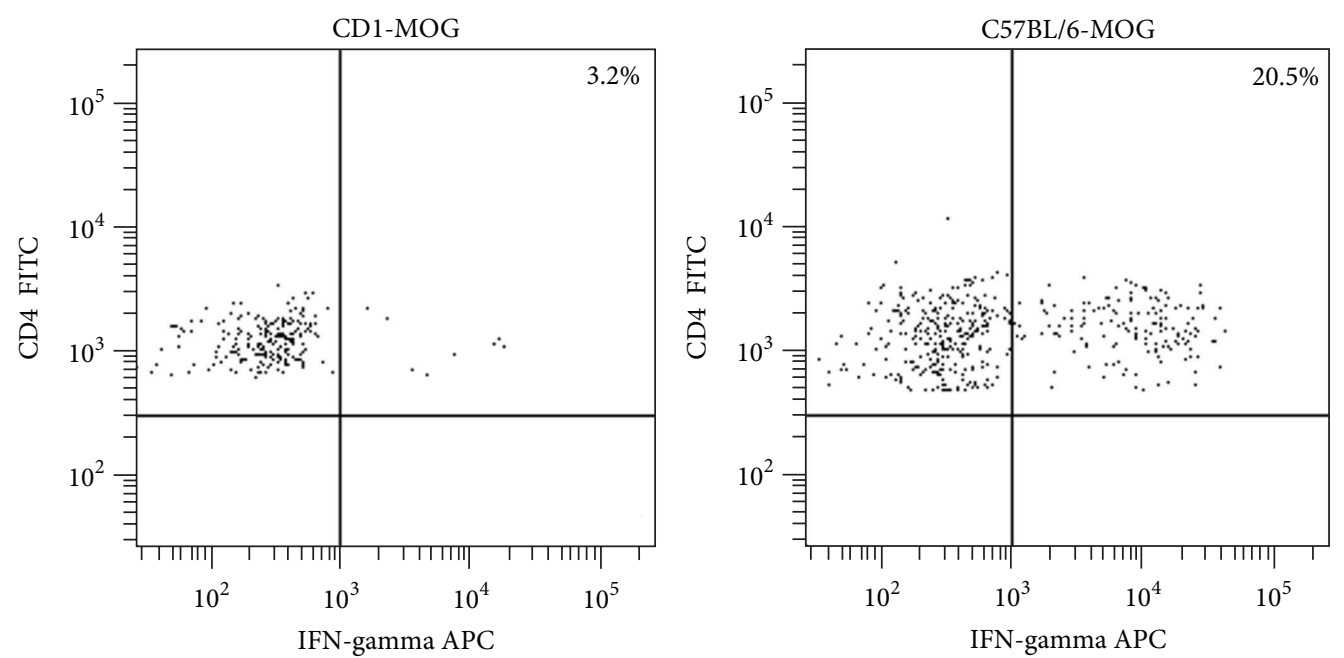

(b)

FIGURE 4: Intracellular production of interferon-gamma (IFN-gamma) by spleen CD4+ T cells. We analyzed these cells in series of seven mice per condition. (a) Splenocytes from C57BL/6 and CD1 mice were cultured in presence of phorbol-12-myristate-13-acetate (PMA) and ionomycin during four hours, and the percentage of CD4+ T cells showing intracellular production of IFN-gamma was analyzed by flow cytometry. We studied nonimmunized mice (NI) and mice immunized with $\mathrm{MOG}_{35-55}$ (MOG) and with PLP $139-151$ (PLP). C57BL/6 mice that developed EAE after immunization with $\mathrm{MOG}_{35-55}$ experienced a significant increase in the percentage of CD4+ T cells producing IFN-gamma. (b) Representative dot plots of IFN-gamma intracellular staining on CD4+ T splenocytes from CD1 and C57BL/6 mice upon immunization with $\mathrm{MOG}_{35-55}$.

beneficial or detrimental effects in demyelinating diseases [20, 21]. In MOG-induced EAE model they are not related to the initiation of the disease and it remains controversial if they can influence disease severity $[22,23]$. We tested the presence of anti-MOG ${ }_{35-55} \operatorname{IgM}$ and IgG antibodies in serum of resistant and susceptible mice. There were no significant increases in anti- $\mathrm{MOG}_{35-55}$ IgG or IgM responses in $\mathrm{MOG}_{35-55}$-immunized mice of either susceptible or resistant strains. This shows that IgM or IgG anti-MOG ${ }_{35-55}$ antibodies do not play a role in inducing susceptibility to $\mathrm{MOG}_{35-55^{-}}$ induced EAE.
We next explored different spleen lymphocyte subsets. Previous reports showed that $\mathrm{CD} 4+\mathrm{T}$ cells are required for the initial induction of progressive EAE and that the disease is downregulated by CD8+ $\mathrm{T}$ cells [24]. Here, we did not find differences in total CD4+ and CD8+ T cell percentages between any of the groups of resistant and susceptible mice. To further discriminate different $\mathrm{T}$ cell responses, we analyzed effector Thl and Th17 subsets. It has been reported that IL-17 impairs the integrity of the blood brain barrier in EAE [25] and that a rise of spleen Th17 cells prolongs the disease [26]. Moreover, a decrease in IL-17 levels 


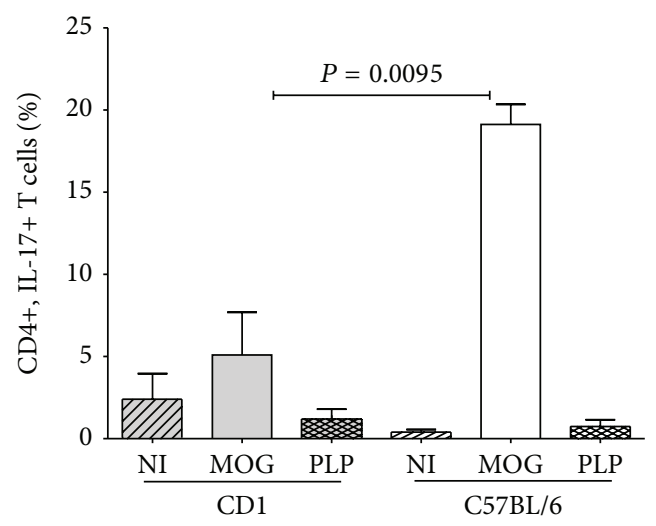

(a)
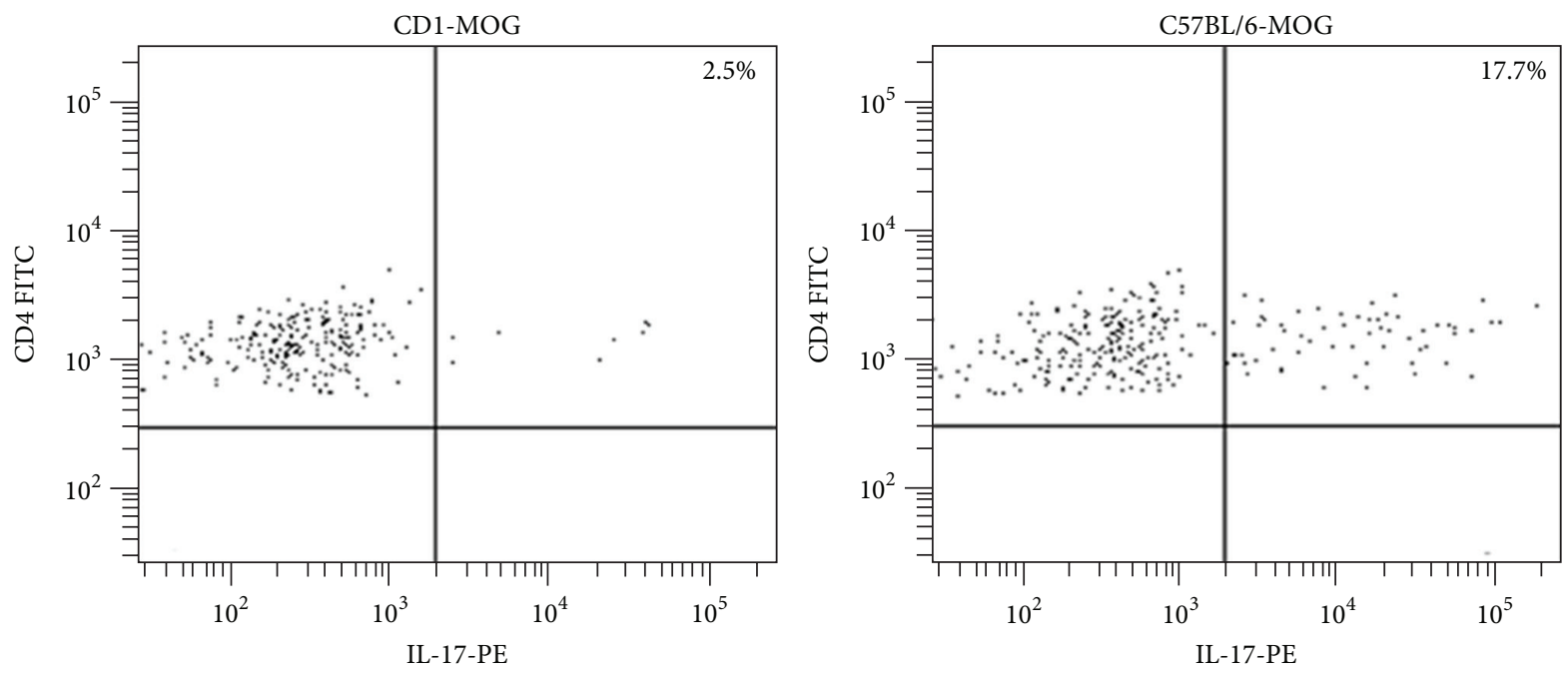

(b)

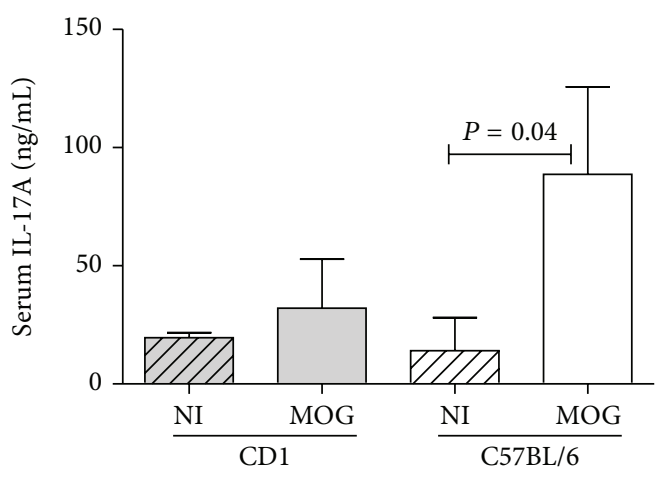

(c)

FIGURE 5: (a) Intracellular production of interleukin-17 (IL-17) by spleen CD4+ T cells. We analyzed these cells in series of seven mice per condition. Splenocytes from C57BL/6 and CD1 mice were cultured in presence of phorbol-12-myristate-13-acetate (PMA) and ionomycin during four hours and the percentage of $\mathrm{CD} 4+\mathrm{T}$ cells showing intracellular production of IL-17 was analyzed by flow cytometry. We studied nonimmunized mice (NI) and mice immunized with $\mathrm{MOG}_{35-55}$ (MOG) and with $\mathrm{PLP}_{139-151}$ (PLP). Only C57BL/6 mice that developed EAE after immunization with $\mathrm{MOG}_{35-55}$ displayed a significant increase in the percentage of CD4+ T cells producing IL-17. (b) Representative dot plots of IL-17 intracellular production by CD4+ T cells from $\mathrm{MOG}_{35-55}$-immunized CD1 and C57BL/6 mice are shown. (c) Serum concentrations of IL-17A in nonimmunized mice (NI) and mice immunized with $\mathrm{MOG}_{35-55}$ (MOG). 
in supernatants of $\mathrm{MOG}_{35-55}$-activated splenocytes runs in parallel with reduced demyelination and axonal damage in mice with EAE [27]. We did not find significant differences in the percentages of CD4+ cells secreting IFN-gamma or IL17 in nonimmunized mice. Conversely, we observed that susceptible mice that developed $\mathrm{EAE}$ in response to $\mathrm{MOG}_{35-55}$ showed significant increases in the secretion of IFN-gamma and IL-17. By contrast, resistant mice were unable to mount any of these responses after the same immunization protocol. In addition, serum levels of IL-17 were increased in susceptible mice immunized with $\mathrm{MOG}_{35-55}$. These data confirm the importance of the induction of Th1/Th17 responses in MOGinduced EAE.

In conclusion, our data show that upregulation of B and, to a lesser extent, of Treg, is closely associated with MHCindependent resistance to MOG-induced EAE in CD1 mice and with the abrogation of both Th1 and Th17 responses, which have a critical role in the development of the disease. These data help to ascertain the regulatory mechanisms than can downmodulate inflammatory responses in demyelinating diseases.

\section{Conflict of Interests}

The authors declare that there is no conflict of interests regarding the publication of this paper.

\section{Acknowledgments}

This study was supported by PS09/01652 and PS09/01338 Grants from Fondo de Investigación Sanitaria (FIS), Instituto de Salud Carlos III, the Ministry of Economy and Competitiveness, Spain, SAF2010/17501 from the Ministry of Science and Innovation, Spain, and "Ajuts per donar Suport als Grups de Recerca de Catalunya (2009 SGR 0793)," sponsored by the "Agència de Gestió d'Ajuts Universitaris i de Recerca" (AGAUR), Generalitat de Catalunya, Spain. Herena Eixarch is supported by the "Sara Borrell" program (CD09/00363), Nieves Marín and Miriam Mecha are supported by Red Española de Esclerosis Múltiple (REEM, RD 07/0060 and RD12/0032), and Carmen Espejo was partially supported by the "Miguel Servet" program (CP07/00146, CPII13/00028), all of them of the FIS, Instituto de Salud Carlos III, Ministry of Economy and Competitiveness, Spain.

\section{References}

[1] The International Multiple Sclerosis Genetics Consortium and The Wellcome Trust Case Control Consortium 2, "Genetic risk and a primary role for cell-mediated immune mechanisms in multiple sclerosis," Nature, vol. 476, pp. 214-219, 2011.

[2] L. I. Levin, K. L. Munger, M. V. Rubertone et al., "Temporal relationship between elevation of Epstein-Barr virus antibody titers and initial onset of neurological symptoms in multiple sclerosis," Journal of the American Medical Association, vol. 293, no. 20, pp. 2496-2500, 2005.

[3] S. Simpson Jr., B. Taylor, L. Blizzard et al., "Higher 25hydroxyvitamin $\mathrm{D}$ is associated with lower relapse risk in multiple sclerosis," Annals of Neurology, vol. 68, no. 2, pp. 193203, 2010.

[4] J. Correale, M. C. Ysrraelit, and M. I. Gaitán, "Immunomodulatory effects of Vitamin D in multiple sclerosis," Brain, vol. 132, no. 5, pp. 1146-1160, 2009.

[5] R. Volchenkov, M. Karlsen, R. Jonsson, and S. Appel, “Type 1 regulatory $\mathrm{T}$ cells and regulatory $\mathrm{B}$ cells induced by tolerogenic dendritic cells," Scandinavian Journal of Immunology, vol. 77, pp. 246-254, 2013.

[6] T. Kinnunen, N. Chamberlain, H. Morbach et al., "Specific peripheral B cell tolerance defects in patients with multiple sclerosis," The Journal of Clinical Investigation, vol. 123, no. 6, pp. 2737-2741, 2013.

[7] J. Correale, M. Farez, and G. Razzitte, "Helminth infections associated with multiple sclerosis induce regulatory B cells," Annals of Neurology, vol. 64, no. 2, pp. 187-199, 2008.

[8] V. S. Ramgolam, Y. Sha, K. L. Marcus et al., "B cells as a therapeutic target for IFN- $\beta$ in relapsing-remitting multiple sclerosis," Journal of Immunology, vol. 186, no. 7, pp. 4518-4526, 2011.

[9] L. Steinman and S. S. Zamvil, "Virtues and pitfalls of EAE for the development of therapies for multiple sclerosis," Trends in Immunology, vol. 26, no. 11, pp. 565-571, 2005.

[10] F. Mokhtarian, D. E. McFarlin, and C. S. Raine, "Adoptive transfer of myelin basic protein-sensitized $\mathrm{T}$ cells produces chronic relapsing demyelinating disease in mice," Nature, vol. 309, no. 5966, pp. 356-358, 1984.

[11] T. Matsushita, K. Yanaba, J.-D. Bouaziz, M. Fujimoto, and T. F. Tedder, "Regulatory B cells inhibit EAE initiation in mice while other B cells promote disease progression," The Journal of Clinical Investigation, vol. 118, no. 10, pp. 3420-3430, 2008.

[12] R. Thomé, A. S. Moraes, A. L. Bombeiro et al., "Chloroquine treatment enhances regulatory $\mathrm{T}$ cells and reduces the severity of experimental autoimmune encephalomyelitis," PLOS ONE, vol. 8, no. 6, Article ID e65913, 2013.

[13] J. Li, X. Zhao, R. Skoff, M. K. Shaw, and H. Y. Tse, "Differential levels of resistance to disease induction and development of relapsing experimental autoimmune encelphalomyelitis in two H-2b-restricted mouse strains," Journal of Neuroimmunology, vol. 234, no. 1-2, pp. 109-114, 2011.

[14] C. Wegner, C. Stadelmann, R. Pförtner et al., "Laquinimod interferes with migratory capacity of T cells and reduces IL-17 levels, inflammatory demyelination and acute axonal damage in mice with experimental autoimmune encephalomyelitis," Journal of Neuroimmunology, vol. 227, no. 1-2, pp. 133-143, 2010.

[15] M. K. Mann, K. Maresz, L. P. Shriver, Y. Tan, and B. N. Dittel, " $\mathrm{B}$ cell regulation of $\mathrm{CD} 4^{+} \mathrm{CD} 25^{+} \mathrm{T}$ regulatory cells and IL-10 via $\mathrm{B} 7$ is essential for recovery from experimental autoimmune encephalomyelitis," Journal of Immunology, vol. 178, no. 6, pp. 3447-3456, 2007.

[16] N. Marín, H. Eixarch, M. J. Mansilla et al., "Anti-myelin antibodies play an important role in the susceptibility to develop PLP-induced EAE," Clinical and Experimental Immunology, 2013.

[17] C. M. Baecher-Allan, C. M. Costantino, G. L. Cvetanovich et al., "CD2 costimulation reveals defective activity by human $\mathrm{CD} 4{ }^{+} \mathrm{CD} 25^{\mathrm{hi}}$ regulatory cells in patients with multiple sclerosis," Journal of Immunology, vol. 186, no. 6, pp. 3317-3326, 2011.

[18] A. Rynda-Apple, E. Huarte, M. Maddaloni, G. Callis, J. A. Skyberg, and D. W. Pascual, "Active immunization using a single dose immunotherapeutic abates established EAE via IL-10 and 
regulatory T cells," European Journal of Immunology, vol. 41, no. 2, pp. 313-323, 2011.

[19] M. B. Fritz and M. L. Zhao, "Active and passive experimental autoimmune encephalomyelitis in strain $129 / \mathrm{J}\left(\mathrm{H}-2^{\mathrm{b}}\right)$ mice," The Journal of Neuroscience Research, vol. 45, pp. 471-474, 1996.

[20] L. M. Villar, M. C. Sádaba, E. Roldán et al., "Intrathecal synthesis of oligoclonal IgM against myelin lipids predicts an aggressive disease course in MS," The Journal of Clinical Investigation, vol. 115, no. 1, pp. 187-194, 2005.

[21] C. Trebst and M. Stangel, "Promotion of remyelination by immunoglobulins: implications for the treatment of multiple sclerosis," Current Pharmaceutical Design, vol. 12, no. 2, pp. 241249, 2006.

[22] S. A. Jagessar, P. A. Smith, E. Blezer et al., "Autoimmunity against myelin oligodendrocyte glycoprotein is dispensable for the initiation although essential for the progression of chronic encephalomyelitis in common marmosets," Journal of Neuropathology and Experimental Neurology, vol. 67, no. 4, pp. 326-340, 2008.

[23] Y. Sekiguchi, M. Ichikawa, M. Takamoto et al., "Antibodies to myelin oligodendrocyte glycoprotein are not involved in the severity of chronic non-remitting experimental autoimmune encephalomyelitis," Immunology Letters, vol. 122, no. 2, pp. 145149, 2009.

[24] E. Montero, G. Nussbaum, J. F. Kaye et al., "Regulation of experimental autoimmune encephalomyelitis by $\mathrm{CD} 4^{+}, \mathrm{CD} 25^{+}$ and $\mathrm{CD}^{+} \mathrm{T}$ cells: analysis using depleting antibodies," Journal of Autoimmunity, vol. 23, no. 1, pp. 1-7, 2004.

[25] J. Huppert, D. Closhen, A. Croxford et al., "Cellular mechanisms of IL-17-induced blood-brain barrier disruption," The FASEB Journal, vol. 24, no. 4, pp. 1023-1034, 2010.

[26] S. Kanakasabai, C. C. Walline, S. Chakraborty, and J. J. Bright, "PPAR $\delta$ deficient mice develop elevated Th1/Th17 responses and prolonged experimental autoimmune encephalomyelitis," Brain Research, vol. 1376, pp. 101-112, 2011.

[27] C. Wegner, C. Stadelmann, R. Pförtner et al., "Laquinimod interferes with migratory capacity of T cells and reduces IL-17 levels, inflammatory demyelination and acute axonal damage in mice with experimental autoimmune encephalomyelitis," Journal of Neuroimmunology, vol. 227, no. 1-2, pp. 133-143, 2010. 


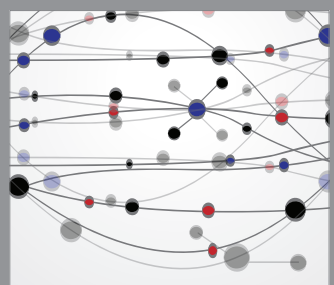

The Scientific World Journal
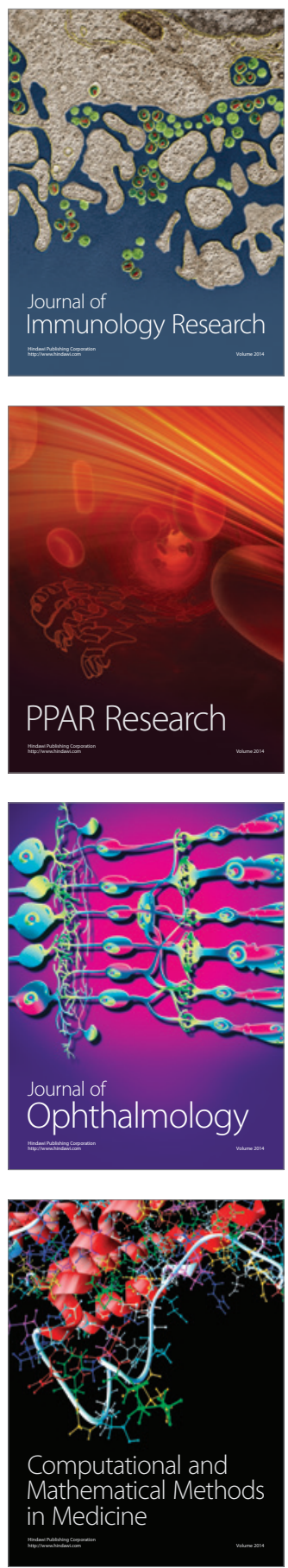

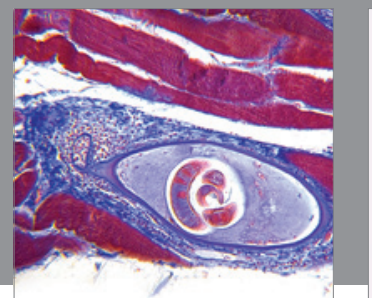

Gastroenterology

Research and Practice
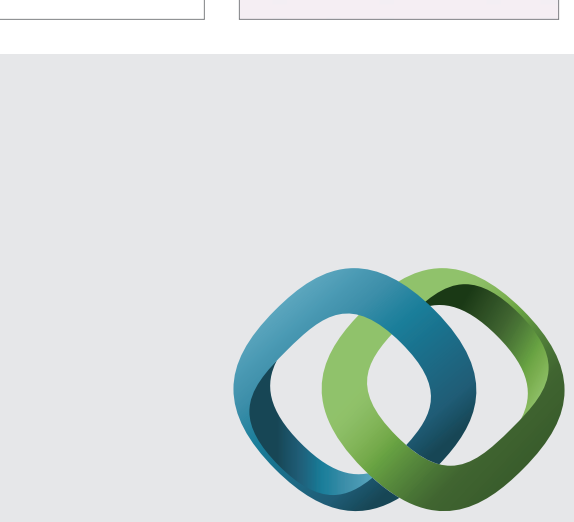

\section{Hindawi}

Submit your manuscripts at

http://www.hindawi.com
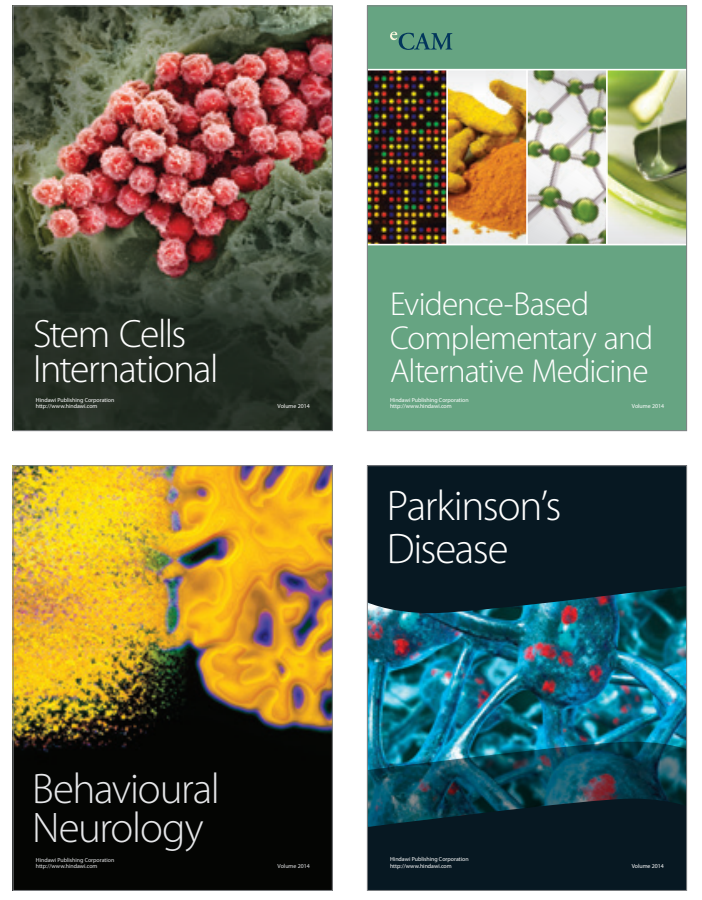
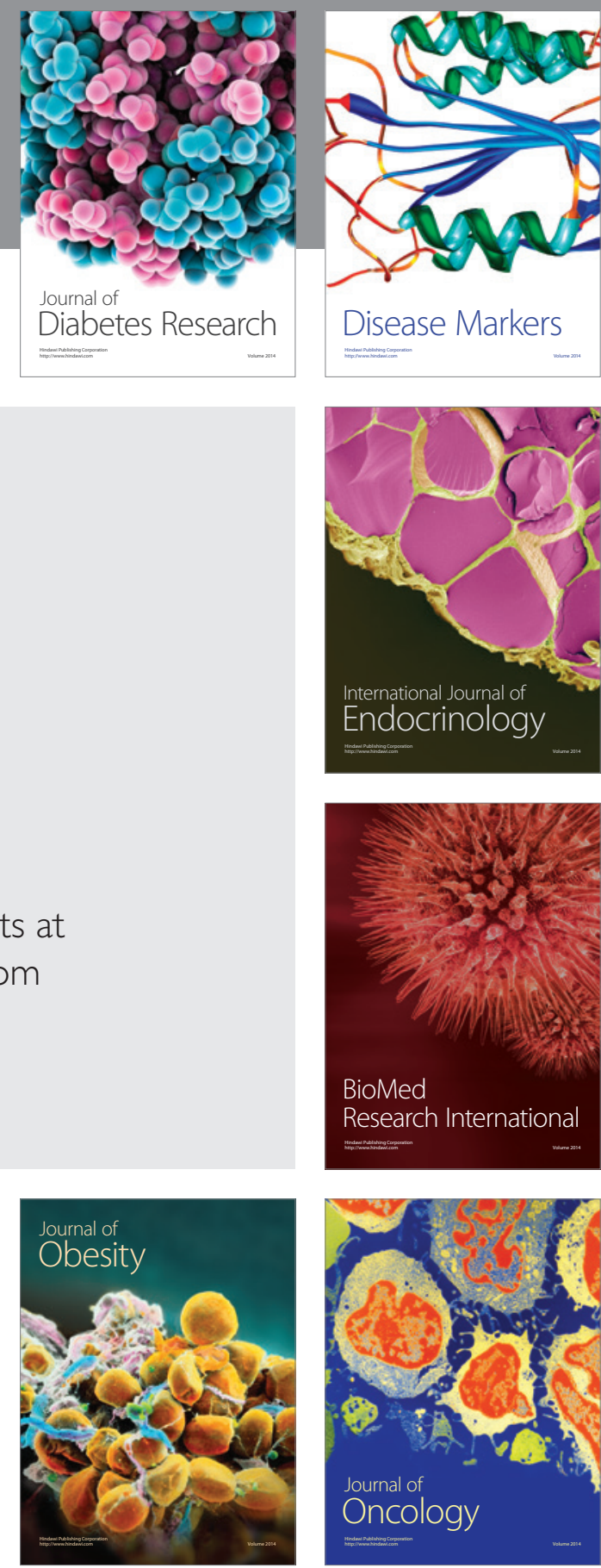

Disease Markers
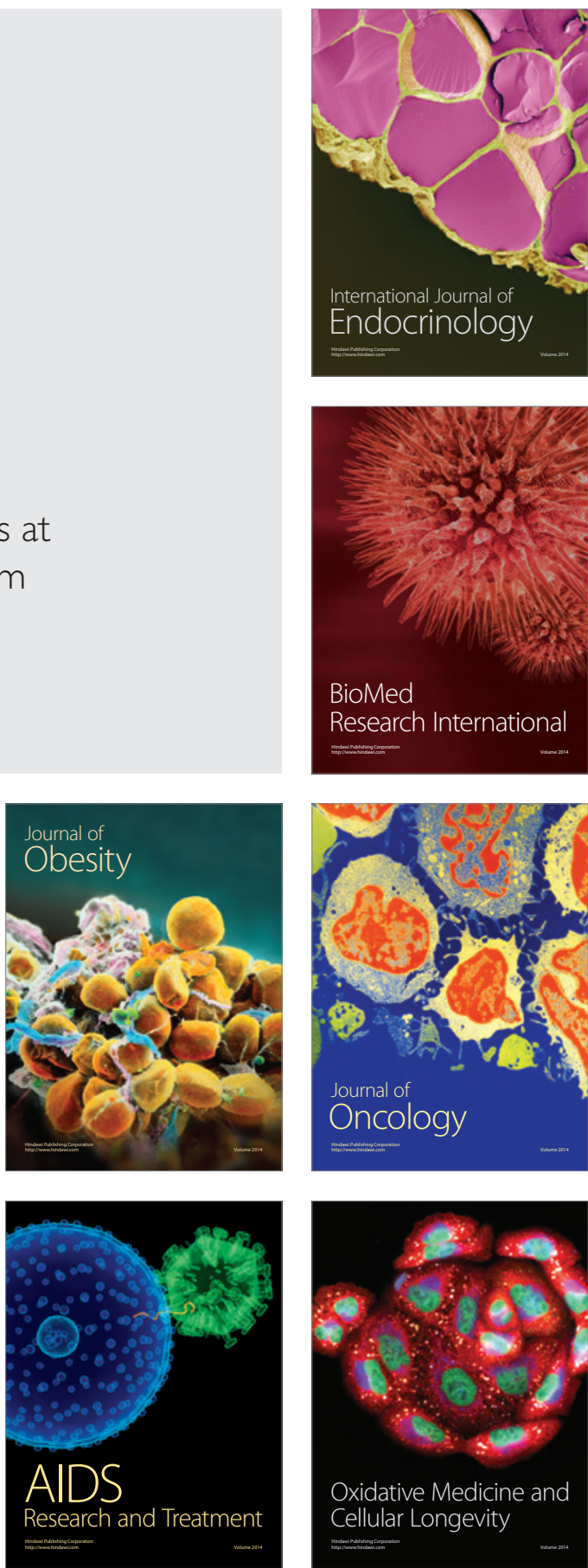